\title{
Pediatric Idiopathic Pulmonary Hemosiderosis Diagnosed by Sputum Analysis: Plain Radiography and Computed Tomography Findings
}

\author{
Ercan Kocakoc ${ }^{a}$ Adem Kiris ${ }^{a}$ Yasar Sen $^{\text {b }}$ Zulkif Bozgeyik ${ }^{a}$ \\ Departments of a Radiology and bediatrics, Faculty of Medicine, Firat University, Elazig, Turkey
}

\section{Key Words}

Idiopathic pulmonary hemosiderosis . Computed tomography $\cdot$ Chest radiography $\cdot$ Hemoptysis

\begin{abstract}
Objective: Idiopathic pulmonary hemosiderosis (IPH) is an uncommon disorder, which is characterized by recurrent hemoptysis, iron deficiency anemia and diffuse parenchymal infiltration on chest radiographs in pediatric patients. We wish to present clinical and radiological (plain radiography and $\mathrm{CT}$ ) findings of this rare pathology. Clinical Presentation and Intervention: A 14-year-old girl was admitted to the pediatric emergency department with complaints of cough, dyspnea, fatigue and bloody sputum for 6 months. She had been hospitalized 3 times during this period and received antibiotics and blood transfusion. Chest $\mathrm{X}$-rays revealed prominent perihilar and bibasilar consolidation. CT showed a ground glass pattern and consolidated areas with increased density. Sputum analysis yielded hemosiderin-laden macrophages. With presumptive diagnosis of IPH, prednisolone was administered. Her symptoms improved on the 5th day of treatment and 1 month later, plain chest radiography demonstrated marked improvement. Conclu-
\end{abstract}

sion: Although IPH is a rare condition, the diagnosis of IPH should be considered, among others, in a patient with hemoptysis and bilateral infiltration in the chest Xray. This may prevent antibiotic misuse and risk of death due to severe hemorrhage.

Copyright $\odot 2003$ S. Karger AG, Basel

\section{Introduction}

Idiopathic pulmonary hemosiderosis (IPH) is an uncommon disorder, which is characterized by recurrent hemoptysis, iron deficiency anemia and diffuse parenchymal infiltration on chest radiographs [1-3]. This disease is usually encountered under age 10 , however it may arise in the second decade and is diagnosed by open lung biopsy [2]. If the biopsy cannot be performed, the clinical presentation of the patient, in the absence of other causes of pulmonary hemorrhage, may establish the diagnosis [3]. We present a case of IPH with typical clinical and radiological findings and hemosiderin-laden macrophages in the sputum.

\section{KARGER \\ Fax + 41613061234 \\ E-Mail karger@karger.ch \\ www. karger.com

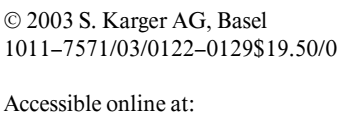

Dr. Ercan Kocakoc

Department of Radiology, Faculty of Medicine

Firat University

TR-23119 Elazig (Turkey)

Tel. +90 42424797 19, Fax +90 42423767 73, E-Mail ekocakoc@hotmail.com 


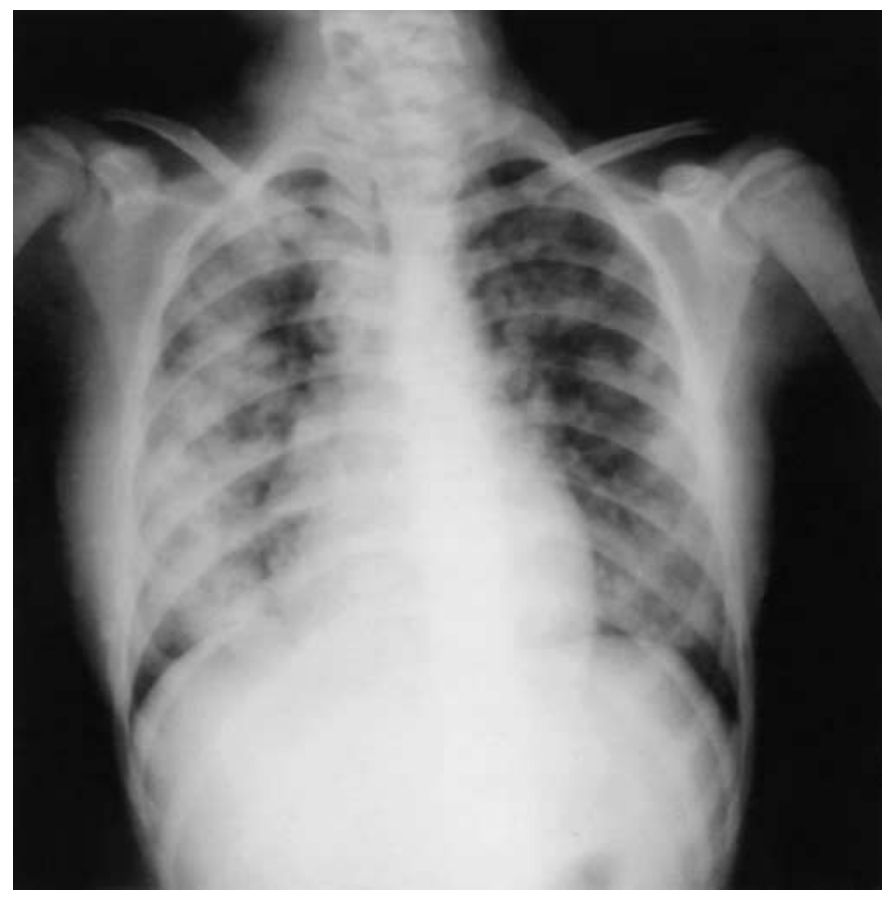

Fig. 1. Posteroanterior chest radiograph showing bilateral air space consolidation areas.

\section{Case Report}

A 14-year-old girl was admitted to the pediatric emergency department with complaints of cough, dyspnea, fatigue and bloody sputum for 6 months. She was hospitalized 3 times during this period and received antibiotics and blood transfusion. She had no family history of lung disease. On physical examination her blood pressure was found to be $110 / 65 \mathrm{~mm} \mathrm{Hg}$. The patient was pale and had tachypnea, tachycardia, crepitating rales and wheezing. Laboratory data included hemoglobin of $9.7 \mathrm{~g} / \mathrm{dl}$, hematocrit of $28 \%$, and MCV of $61.2 \mathrm{fl}$. The platelet count was $380,000 / \mathrm{mm}^{3}$ with iron deficiency anemia. Acute phase reactants and WBC were normal. Sputum culture, PPD test and acid-resistant bacteria tests were negative. Coagulation and bloodurine biochemical tests were within normal limits. The stools were positive for occult blood and sputum analysis yielded hemosiderinladen macrophages elevated 3-fold. Chest X-ray revealed prominent perihilar and bibasilar consolidation (fig. 1). CT (10 mm thickness, $120 \mathrm{kVp}, 200 \mathrm{mAs}$, Hitachi 1000, Tokyo, Japan) showed a ground glass pattern and consolidated areas with increased density as seen in patients with alveolar hemorrhage (fig. 2). The patient refused to undergo an open lung biopsy. Milk radioallorgosorbent test, antineutrophilic cytoplasmic antibody test, rheumatoid factor, anticardiolipin antibody and anti-DNA tests were all negative. Immunoglobulins (IgG, M, A) and complement (C3, C4, CH5O) were normal. Electrocardiography and echocardiography revealed findings of slight right ventricular overload. With presumptive diagnosis of IPH, prednisolone $(2 \mathrm{mg} / \mathrm{kg} / \mathrm{day})$ was administered. The patient's symptoms improved on the 5th day of treatment and she was discharged from the hospital on the 14th day. One month later, plain chest radiography demonstrated marked improvement (fig. 3).
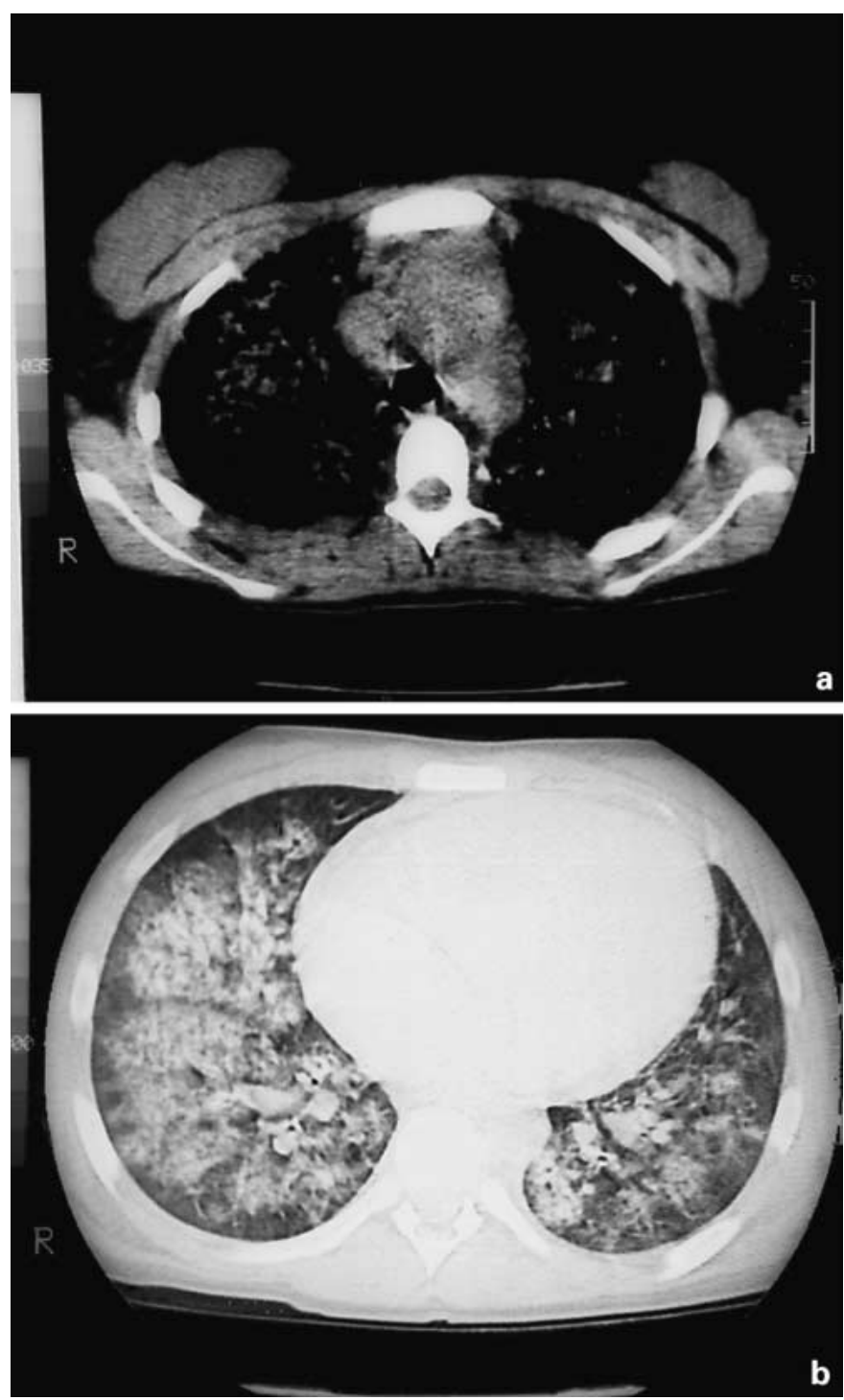

Fig. 2. Contrast-enhanced $\mathrm{CT}(120 \mathrm{kVp}, 200 \mathrm{mAs}$, thickness: $10 \mathrm{~mm}$, normal reconstruction algorithm) of the chest demonstrating bilateral marked air space consolidation and increased density areas supporting alveolar hemorrhage. a Mediastinal window. b Lung window.

\section{Discussion}

Virchow first described the pathologic findings of IPH in 1864 and clinical and radiological diagnosis was described by Waldenstrom in 1944 [cited after ref. 4]. Estimated incidence of this disorder is $0.24-1.23$ per $1,000,000$ population [3]. There is no gender (female:male ratio of 1:1), racial or geographic propensity [5]. Familial tendency has been described [6]. Recurrent multifocal alveolar capillary hemorrhage is responsible for the clini- 


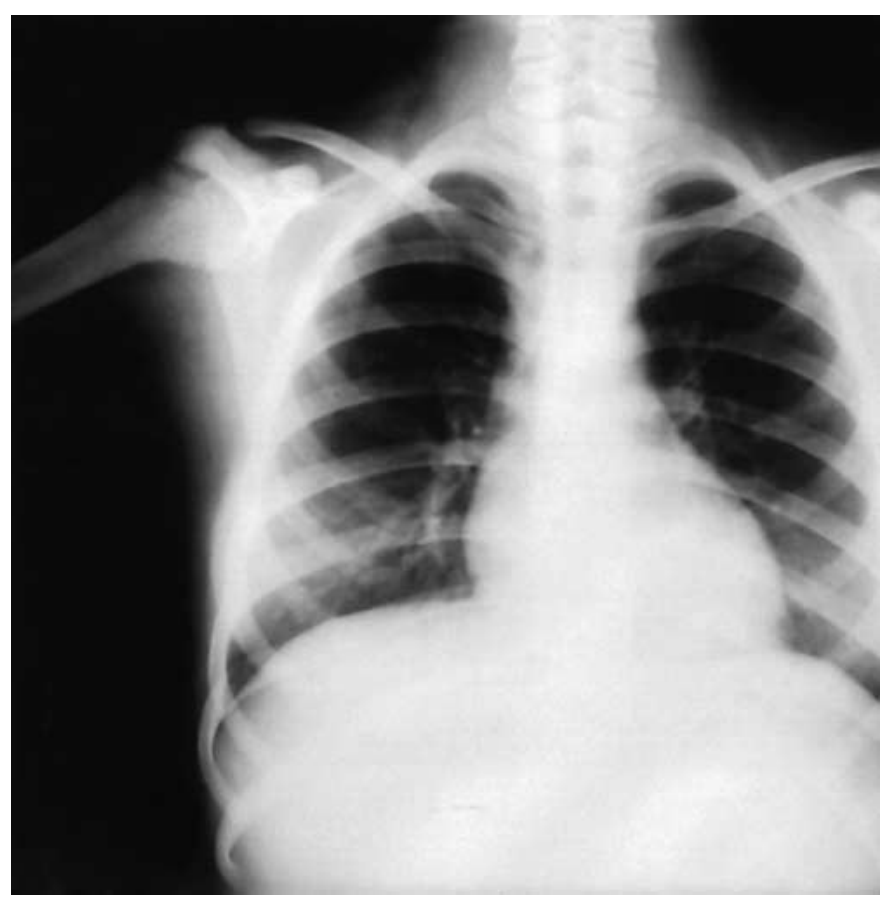

Fig. 3. Follow-up chest radiograph showing marked improvement compared to the previous findings.

cal and laboratory findings [7]. Etiology is obscure but the dramatic response to steroid treatment suggests an immunologic defect in the basal membrane of the pulmonary capillaries [7]. The clinical course may vary but most patients die in the early phase of the disease because of acute pulmonary hemorrhage. However, complete remission can be achieved with early diagnosis and treatment. Some patients may progress to chronic pulmonary fibrosis, chronic respiratory insufficiency, and ultimately pulmonary hypertension [3]. The differential diagnosis includes Goodpasture's syndrome with or without renal involvement, hemosiderosis secondary to vasculitis, pulmonary embolus, pulmonary venous hypertension such as long-standing mitral valvular stenosis, pulmonary vein stenosis and veno-occlusive disease, and bleeding diatheses due to cancer or infections and lymphangioleiomyomatosis [8]. Our case could not be linked to any of these diseases. Therefore, in the absence of a gold standard test, IPH was the diagnosis of choice by exclusion, given the histologic evidence.

Histologically, the unifying features of diffuse pulmonary hemorrhage are the presence of recent hemorrhage in alveolar spaces and hemosiderin-laden macrophages in alveolar spaces and interstitium [2]. The presence of the hemosiderin-laden macrophages in the sputum reflects the presence of pulmonary alveolar hemorrhage, but does not reflect any specific cause.

Typical radiographic findings are air space consolidation and ground glass appearance [2]. Perihilar areas and both lung bases are mostly affected [9]. The apexes and costophrenic angles are typically unaffected. However if the parenchymal involvement is extensive, as in our case, costophrenic angles and apexes may also be affected [9]. Consolidation areas may turn to a reticular pattern within a few days that may then be resorbed completely, as in our case, or may advance to fibrosis with recurrent episodes of pulmonary hemorrhage. Lymphadenopathy and pleural effusion are not common [9]. While the sensitivity of CT is superior to that of the plain film in displaying the characteristic acinar pattern [1], the CT findings are nonspecific because pulmonary edema and alveolar hemorrhage or other diffuse air space disorders, all display similar CT changes. Nonetheless, a diffuse dense acinar pattern supports the alveolar nature of the pulmonary pathology [1]. The reason of the increased density in the CT is intraalveolar hemorrhage and/or hemosiderin-laden macrophages. The pattern of acute alveolar hemorrhage may be more easily distinguished by CT [1] than by plain X-ray films, which may provide images that mimic pneumonia and thus result in diagnostic delay and unnecessary antibiotic therapy [10]. Sometimes the source of hemorrhage is extrapulmonary and the blood gets aspirated into the alveoli, as in endobronchial tuberculosis, or the blood may spread from one segment of the lung to another or from one to the contralateral side $[11,12]$.

Corticosteroids are the initial treatment of choice. If the steroids are ineffective, cytotoxic drugs may be used. Long-term prognosis of IPH is poor and most patients develop pulmonary fibrosis 5 years after the initial diagnosis [7].

\section{Conclusion}

Although IPH is a rare condition, in a patient with hemoptysis and bilateral scattered air space disease in the chest X-ray, the diagnosis of IPH should be considered, among others. This may prevent antibiotic misuse and risk of death due to severe hemorrhage.

Med Princ Pract 2003;12:129-132 


\section{References}

1 Akyar S, Ozbek SS: Computed tomography findings in idiopathic pulmonary hemosiderosis. Respiration 1993;60:63-64.

2 Primack SL, Miller RR, Muller NL: Diffuse pulmonary hemorrhage: Clinical, pathologic, and imaging features. AJR Am J Roentgenol 1995; 164:295-300.

3 Saeed MM, Woo MS, MacLaughlin EF, Margetis MF, Keens TG: Prognosis in pediatric idiopathic pulmonary hemosiderosis. Chest 1999 ; 116:721-725.

4 Heiner DC: Pulmonary hemosiderosis; in Chernick V, Kendig EL Jr (eds): Disorders of the Respiratory Tract in Children. Philadelphia, Saunders, 1990, pp 498-509.
5 Le Clainche L, Bourgeois ML, Fauroux B, Forenza N, Dommergues JP, Desbois JC, Bellon G, Derelle J, Dutau G, Marguet C, Pin I, TillieLeblond I, Scheinmann P, De Blic J: Long-term outcome of idiopathic pulmonary hemosiderosis in children. Medicine 2000;79:318-326.

6 Kiper N, Gocmen A, Ozcelik U, Dilber E, Anadol D: Long-term clinical course of patient with idiopathic pulmonary hemosiderosis (1979-1994): Prolonged survival with low-dose corticosteroid therapy. Pediatr Pulmonol 1999; 27:180-184.

7 Soergel KH, Sommers SC: Idiopathic pulmonary hemosiderosis and related syndromes. Am J Med 1962;32:499-511.

8 Le Blanche AF, Pascaud JL, Bouillet P, Majouret JF, Provensol T, Gaillard S: Pulmonary hemosiderosis: Hemodynamic or idiopathic origin? Diagnostic contribution of imaging and review of the literature. J Radiol 1991;72:3541.
9 Bronson SM: Idiopathic pulmonary hemosiderosis in adults: Report of a case and review of the literature. AJR Am J Roentgenol 1960;83: 260-273.

10 Paul G, Bhatnagar SK, Maskary SA, Kuruvilla S, Kurien S: Idiopathic pulmonary hemosiderosis. J Trop Pediatr 2000;46:243-245.

11 Abal AT, Nair PC, Cherian J: Haemoptysis: Aetiology, evaluation and outcome: A prospective study in a third-world country. Respir Med 2001;95:548-552.

12 Volckaert A, Roels P, Van der Niepen P, Schandevyl W: Endobronchial tuberculosis: Report of three cases. Eur J Respir Dis 1987; 70:99-101. 